\title{
Archéologie de la « Petite histoire de la photographie »
}

André Gunthert

\section{(2) OpenEdition}

12 Journals

Édition électronique

URL : http://journals.openedition.org/imagesrevues/292

DOI : 10.4000/imagesrevues.292

ISSN : 1778-3801

Éditeur :

Centre d'Histoire et Théorie des Arts, Groupe d'Anthropologie Historique de l'Occident Médiéval, Laboratoire d'Anthropologie Sociale, UMR 8210 Anthropologie et Histoire des Mondes Antiques

\section{Référence électronique}

André Gunthert, "Archéologie de la « Petite histoire de la photographie » », Images Re-vues [En ligne], Hors-série 2 | 2010, document 7, mis en ligne le 01 janvier 2010, consulté le 03 février 2021. URL http://journals.openedition.org/imagesrevues/292 ; DOI : https://doi.org/10.4000/imagesrevues.292

Ce document a été généré automatiquement le 3 février 2021.

Images Re-vues est mise à disposition selon les termes de la Licence Creative Commons Attribution Pas d'Utilisation Commerciale 4.0 International. 


\title{
Archéologie de la « Petite histoire de la photographie »
}

\author{
André Gunthert
}

La « Petite histoire de la photographie » a parfois dérouté par sa «nébulosité » . Pour l'expliquer, on a cru pouvoir lire derrière son titre le choix d'un conte et interpréter son déroulement comme un parcours d'images, à la manière d'une projection de diapositives ${ }^{2 .}$ Il s'agit en effet d'un texte à bien des égards étrange - pourtant, le cadre dans lequel il s'inscrit impose des contraintes à la fois plus simples et plus précises. À la différence de L'Fuvre d'art, essai publié dans une revue scientifique, la " Petite histoire » est un article rédigé pour un magazine culturel, Die Literarische Welt, dont Benjamin est un collaborateur régulier depuis 1925. Ce contexte impose d'emblée certaines

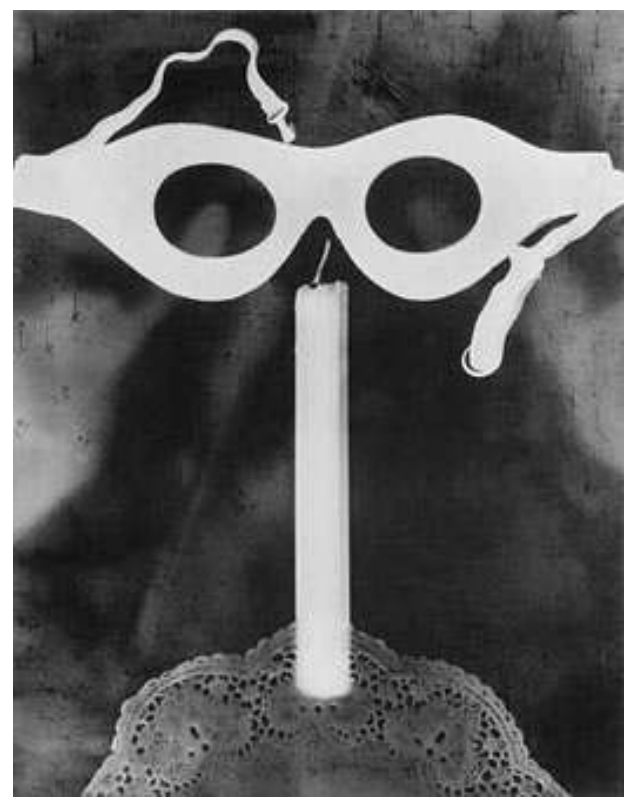
déterminations, qui forment autant d'indications de lecture. S'adressant à un public certes lettré, mais probablement peu au fait de l'histoire de la photographie, un texte de ce type se doit de proposer à la fois une vulgarisation et une synthèse - ce que traduit rigoureusement son titre. Mais surtout, les règles du journalisme culturel supposent d'évaluer la légitimité d'un article à son rapport à l'actualité. Or, signée par celui que les lecteurs de la revue connaissent pour ses comptes rendus littéraires, la « Petite histoire » doit d'abord son existence à une remarquable conjonction éditoriale : la parution d'un recueil d'images consacré à la photographie ancienne, premier du genre, par les collectionneurs Helmuth Bossert et Heinrich Guttmann $(1930)^{3}$, suivi à brève échéance par la publication de deux autres 
albums : le premier dédié à Eugène Atget, par Camille Recht (1930), le second à David Octavius Hill, par le critique Heinrich Schwarz (1931) ${ }^{4}$.

Pour mesurer ce que cette conjonction a d'exceptionnel, il faut se souvenir qu'à ce moment, les ouvrages en langue allemande consacrés à l'histoire de la photographie se comptent sur les doigts d'une main ${ }^{5}$. Déjà ancienne, la Geschichte der photographie (1905) de l'Autrichien Josef-Maria Eder reste un ouvrage de référence largement mis à profit par les spécialistes, tandis que l'historien berlinois Erich Stenger publie en 1929 un petit catalogue à l'occasion d'une discrète exposition du Deutsches Museum 6 . Pourtant, la renommée de ces travaux ne dépasse pas un cercle très restreint : de façon significative, Benjamin n'y fait aucune allusion et il semble bien qu'il n'ait eu recours à aucun de ces deux ouvrages. L'érudition documentaire n'est pas ce qui a éveillé son intérêt : à la différence de ces travaux, arides chronologies des innovations techniques, peu ou pas illustrés, les trois recueils en question laissent délibérément la première place à l'image. Faisant œuvre pionnière dans ce qui s'imposera rapidement comme la nouvelle direction de l'historiographie photographique ${ }^{7}$, ils font découvrir pour la première fois des clichés auquel le grand public n'avait jusqu'alors eu aucun accès. Rien d'étonnant à ce qu'un grand magazine culturel comme Die Literarische Welt consacre trois livraisons à ce sujet aussi nouveau qu'intéressant ${ }^{8}$ (il faut souligner que la longueur de l'article de Benjamin, qui sera publié dans trois numéros successifs, est inhabituelle pour la Literarische Welt, publication hebdomadaire d'environ huit pages ; la «Petite histoire » constitue donc l'un des articles les plus longs jamais publiés dans ses colonnes).

Outre ces ouvrages historiques, scrupuleusement cités en note, l'article de Benjamin s'appuie également sur trois autres parutions récentes : les albums des photographes Albert Renger-Patzsch (1928), Karl Blossfeldt (1928) et August Sander (1929) ${ }^{9}$. Là encore, le rapprochement de ces publications témoigne de la vitalité du champ photographique au tournant des années 1930, cette fois sous l'angle de la création contemporaine. Après le long tunnel du pictorialisme, la vigueur des nouveaux supports de l'après-guerre presse et publicité illustrées -, mais aussi de nouveaux questionnements esthétiques, dans le sillage du surréalisme ou du Bauhaus, produisent en l'espace de quelques années un renouvellement considérable de l'iconographie et des problématiques photographiques ${ }^{10}$. C'est dans ce cadre que les artistes, les écrivains ou les philosophes commencent à s'intéresser à ce médium. Après le coup d'envoi constitué par le célèbre Peinture, photographie, film (1925) de László Moholy-Nagy ${ }^{11}$, le public cultivé voit se succéder les contributions de Siegfried Kracauer, Bertolt Brecht, Thomas Mann, Alfred Döblin et quelques autres ${ }^{12}$. Walter Benjamin figure donc parmi les premiers intellectuels d'envergure à aborder sérieusement la question de la photographie ${ }^{13}$.

Une fois posé l'effet de mode de la fin des années 1920, reste à savoir comment et pourquoi un philosophe, un littéraire qui ignore à peu près tout des problématiques de la technique aborde la photographie. L'une des clefs essentielle est fournie dès la première phrase de la Petite histoire ${ }^{14}$ : il s'agit du modèle de l'imprimerie, archétype d'une structure de démocratisation d'un accès au savoir par le biais de la multiplication mécanique, particulièrement pregnant en Allemagne, que Benjamin reprend de Schwartz et que l'on retrouve dans plusieurs autres ouvrages d'histoire de la photographie de l'époque ${ }^{15}$. Ceux-ci empruntent à l'historiographie de l'imprimerie un cadre de description vaguement wébérien qui décrit l'invention comme s'inscrivant dans l'espace d'une nécessité historico-sociologique (au demeurant mal définie ${ }^{16}$ ). 
Quelles que soient les incertitudes de ce modèle, celui-ci demeure incontestablement la première tentative de penser les problématiques techniques de l'invention, de la multiplication ou d'un accès facilité, aisément transposable à la photographie.

La bibliographie de Benjamin comporte trois textes qui manifestent son intérêt pour la photographie antérieurement à la rédaction de la " Petite histoire ». Le premier, qui date de 1924, constitue un cas très particulier et tout à fait passionnant : il s'agit de la traduction en allemand par Benjamin, pour la revue d'avant-garde $G$, de la préface rédigée par Tristan Tzara pour l'album de photogrammes de Man Ray, paru en 1922, intitulé Champs délicieux ${ }^{17}$. Le deuxième est un court texte non publié, daté de 1925, dans lequel Benjamin réagit vivement à une prise de position " conservatrice " d'un rédacteur de la Literarische Welt contre les journaux illustrés ${ }^{18}$. Enfin, le troisième est une note de lecture de 1928 saluant la parution de l'ouvrage de Karl Blossfeldt, Urformen der Kunst ${ }^{19}$, dont plusieurs éléments seront répris dans la " Petite histoire ». Deux remarques s'imposent : premièrement, l'accès de Benjamin à la photographie s'inscrit dans un contexte résolument avant-gardiste, à l'écart des sentiers battus - voire (pour le texte de Tzara) dans un cadre de négation de l'esthétique ou de l'art au sens traditionnel ; deuxièmement, si on met en balance ses analyses avec d'autres interventions contemporaines d'écrivains sur la photographie comme Thomas Mann ou Georges Bataille, il faut reconnaître à Benjamin une indéniable perspicacité et un réel discernement en ce qui concerne des problématiques techniques. Sur l'ouvrage de Blossfeldt, par exemple, alors que Bataille demeure sur le terrain du référent, prenant prétexte des images pour élaborer une sémiologie du langage des fleurs ${ }^{20}$, Benjamin, s'appuyant sur les mécanismes de la caricature, produit une glose brillante sur la problématique de l'agrandissement.

Malgré ces quelques coups d'essai, la « Petite Histoire » constitue bel et bien le travail par lequel Benjamin affronte, sinon découvre, la question photographique. Loin d'être un spécialiste de l'histoire du médium, le philosophe, à l'instar d'un journaliste, s'appuie ici sur une documentation limitée, de seconde main ${ }^{21}$, dans laquelle il puise avec libéralité : le genre auquel appartient l'article, celui du compte rendu, explique que sa principale caractéristique soit une économie d'écriture essentiellement basée sur l'emprunt. La plupart des matériaux utilisés par Benjamin proviennent de lectures récentes, devant servir à la rédaction de son texte. Parmi les trois recueils d'histoire de la photographie, Bossert et Guttmann ou Schwarz comportent des indications bibliographiques mises à profit par Benjamin pour compléter son information historique : quatre autres ouvrages plus anciens seront consultés, en particulier une préface de 1907 de l'historien de l'art Alfred Lichtwark, largement exploitée, à laquelle est notamment empruntée la fameuse thèse de la démocratisation du portrait (reprise quelques années plus tard par Gisèle Freund) ${ }^{22}$. La part des lectures personnelles de Benjamin utilisées dans la " Petite Histoire » apparaît peu importante, à l'exception du Salon de 1859 de Baudelaire et des textes de Moholy-Nagy ${ }^{23}$. Au total, si l'on y ajoute les trois albums de photographie contemporaine précédemment cités, à peine une dizaine d'ouvrages auront été consultés pour sa rédaction. Compte tenu de leur exploitation souvent abusive $e^{24}$, c'est trop peu pour répondre aux critères de l'écriture académique, mais évidemment beaucoup pour un simple article de journal. Cette disproportion dévoile l'intérêt immédiat de Benjamin pour le sujet, que des recherches ultérieures viendront confirmer ${ }^{25}$. Malgré la mauvaise qualité de l'information historique dont il dispose et le caractère très partiel de l'iconographie dont il a pris connaissance, la 
rencontre de la photographie ancienne lui aura ouvert le chemin d'une interrogation essentielle du rapport à l'histoire, de l'accès au passé.

Pour un article à caractère historique, fût-il de vulgarisation, la " Petite histoire " commence d'une façon curieuse. Plaçant la question de l'origine de la photographie sous le signe du "brouillard » (Nebel), le texte réussit le tour de force de déployer près d'une centaine de lignes avant de fournir une première date précise ${ }^{26}$. Malgré l'accumulation de déictiques à caractère temporel (« Lorsque ce résultat, après environ cinq ans d'efforts, fut accordé en même temps à Niepce et Daguerre ", " Pendant des décennies ", « la première décennie [de la photographie] $»^{27}$, etc.), le texte, oubliant de préciser ces indications par un millésime, perd son lecteur dans un paysage aux contours flous, une narration qui possède toutes les apparences de l'histoire, mais risque fort de n'en être qu'un simulacre.

Il est souvent irritant de voir les exégètes de Benjamin prendre au sérieux ses approximations historiques ou ses chronologies incertaines. L'« âge d'or » (Blüte) supposé baigner la première décennie de la photographie et illustré par les noms de Hill, Cameron, Hugo ou Nadar ne correspond à aucune réalité historique, pas plus que la prétendue " industrialisation » (Industrialisierung) qui aurait immédiatement suivi cette période. L'époque à laquelle ses exemples pourrait renvoyer recouvrirait grossièrement les années 1850-1860, soit la deuxième (et non la première) décennie après la divulgation du daguerréotype. Toutefois, David-Octavius Hill (et Robert Adamson) ayant pratiqué la photographie entre 1843 et 1847, Charles-Victor Hugo en 1852, Nadar à partir de 1854 et Julia Margaret Cameron à partir de 1860, il est impossible de réunir leurs travaux sous la même décade. Quant à André-Adolphe Disdéri, allusivement donné pour le représentant de l'industrialisation du médium avec sa fameuse carte de visite photographique, il en dépose en réalité le brevet en 1854, l'année même des débuts de Nadar.

9 La relative imprécision dont témoignent certaines des sources de l'article (en particulier Bossert et Guttmann) suffit-elle à expliquer ces tâtonnements ? Compte tenu de celles qui présentent au contraire une chronologie correcte (en particulier Schwarz), il est difficile de ne pas admettre qu'un peu d'attention aurait suffi à rétablir une description plus exacte. En réalité, la confusion entretenue par Benjamin forme la condition nécessaire à l'adoption du schéma sur lequel repose la " Petite histoire » : l'opposition fantasmatique d'une longue décadence qui repousse dans des contreforts d'autant plus lointains l'âge d'or supposé de la pratique photographique.

Replacée dans son contexte, cette fiction historique est moins extravagante qu'il y paraît. Portraits d'atelier traditionnels ou recherches pictorialistes, les produits de l'activité photographique d'avant-guerre présentaient encore une incontestable proximité avec la photographie du XIX ${ }^{e}$ siècle. Bousculant la relative stabilité de cette iconographie, les nouvelles images des années 1920 repoussent bel et bien la photographie ancienne dans un passé qui paraît d'un coup plus reculé. Cet écart nouveau a probablement joué un rôle majeur dans le regain d'intérêt pour l'histoire de la photographie, et dans le fait que l'angle iconographique prenne désormais le pas sur la description technicienne. Cette évolution de la réception (dont on trouve également trace chez Moholy-Nagy), la « Petite histoire » la traduit à sa façon par ce qui constitue une véritable stratégie de l'éloignement.

11 À quoi correspond cette stratégie ? Si l'on fait la part des multiples emprunts ou reformulations du texte, celui-ci ne propose peut-être qu'une seule idée véritablement 
originale, mais elle est fondamentale : l'idée que la photographie, au-delà de la simple représentation, donne accès à l'être même, voire au secret de l'être, dans ce qu'il a de plus intime (avec sa déclinaison sur le plan esthétique : le dépassement de l'art opéré par la photographie). Développée dès le début du texte, cette thèse s'appuie sur deux images effectivement reproduites dans Die Literarische Welt : la première, un portrait de femme réalisé par D. $O$. Hill et R. Adamson vers 1843 , tiré de $S c h w a r z^{28}$ et décrit en ces termes:
«La photographie nous confronte à quelque chose de nouveau et de singulier : dans cette marchande de poisson de Newhaven, qui baisse les yeux au sol avec une pudeur si nonchalante, si séduisante, il reste quelque chose qui ne se réduit pas au témoignage de l'art de Hill, quelque chose qu'on ne soumettra pas au silence, qui réclame insolemment le nom de celle qui a vécu là, mais aussi de celle qui est encore vraiment là et ne se laissera jamais complètement absorber dans l' "art". " ${ }^{29}$

Étant donné la démonstration qu'elle illustre, une image aussi peu spontanée qu'un portrait de Hill et Adamson, commentée par Benjamin comme s'il s'agissait d'un instantané, ne représentait pas forcément le choix le plus judicieux. Mais la deuxième photographie, portrait d'un couple dont la légende nous indique qu'il représente Karl Dauthendey et sa fiancée en 1857, puisée dans Bossert et Guttmann ${ }^{30}$, présente un cas de figure encore plus intéressant :

«Ou bien l'on découvre l'image de Dauthendey, le photographe, père du poète, à l'époque de ses fiançailles avec la femme qu'il trouva un jour, peu après la naissance de son sixième enfant, les veines tranchées dans la chambre à coucher de sa maison de Moscou. On la voit ici à côté de lui, on dirait qu'il la soutient, mais son regard à elle est fixé au-delà de lui, comme aspiré vers des lointains funestes. Si l'on s'est plongé assez longtemps dans une telle image, on aperçoit combien, ici aussi, les contraires se touchent : la plus exacte technique peut donner à ses produits une valeur magique, beaucoup plus que celle dont pourrait jouir à nos yeux une image peinte. Malgré toute l'ingéniosité du photographe, malgré l'affectation de l'attitude de son modèle, le spectateur ressent le besoin irrésistible de chercher dans une telle image la plus petite étincelle de hasard, d'ici et maintenant, grâce à quoi la réalité a pour ainsi dire brûlé de part en part le caractère d'image - le besoin de trouver l'endroit invisible où, dans l'apparence de cette minute depuis longtemps écoulée, niche aujourd'hui encore l'avenir, et si éloquemment que, regardant en arrière, nous pouvons le découvrir. $»^{31}$

13 Où Benjamin aperçoit-il, dans ce très banal portrait d'atelier, les éléments d'une telle tragédie ? L'information biographique complaisamment déployée provient du livre de souvenirs consacré par le poète Max Dauthendey à son père Karl, l'un des premiers Allemands à s'essayer au daguerréotype dans les années 1840. Cet ouvrage occupe dans le paysage éditorial outre-Rhin une position comparable au Quand j'étais photographe de Nadar, et se trouve constamment cité par les histoires du médium - y compris par Bossert et Guttmann qui y puisent nombre d'informations plus ou moins anecdotiques, et ont probablement été ravis de découvrir le portrait du couple, que Benjamin reproduit à son tour.

14 Ce dernier a également consulté l'ouvrage de Dauthendey et y découvre l'épisode dramatique du suicide, relaté en quelques lignes ${ }^{32}$. Malgré la brièveté de la narration, on peut y reconnaître les signes cliniques d'un cas extrême de dépression postnatale ${ }^{33}$ ce qui n'aurait guère d'importance, n'était le caractère imprévisible et inexplicable d'un tel acte. C'est ainsi qu'en lieu et place de l'exposé attendu des causes du suicide, le récit enchaîne sur la description d'une photographie plus ancienne du jeune couple, conservée par la demi-sœur de Max, et commentée en ces termes : « On ne voit encore 
aucune trace du malheur futur sur cette image, sinon que le mâle regard de mon père, sombre et appuyé, trahit une brutalité juvénile, susceptible de blesser cette femme qui le contemple attentivement. $\|^{34}$

En dehors du motif de la prophétie rétrospective que Benjamin reprend à sa manière dans sa propre narration, il est clair que c'est ici l'économie du récit qui nourrit la lecture de l'image, au point de lui imposer un sens que celle-ci ne comporte en aucune façon. Benjamin nous en offre la preuve dans une méprise qui a tout du lapsus. Dans l'ouvrage de Dauthendey, l'épisode du suicide est daté de 1855. L'écart de deux ans avec l'indication fournie par la légende de Bossert et Guttmann aurait pu attirer l'attention de Benjamin. Emporté par la force de la situation narrative, celui-ci superpose le récit d'image de Dauthendey à la reproduction qu'il a sous les yeux, sans s'apercevoir qu'il ne s'agit pas de la même personne : la " fiancée » du portrait de Bossert et Guttmann est la seconde femme de Karl Dauthendey, Mlle Friedrich, qu'il épouse deux ans après le tragique décès de sa première compagne.

Sans même insister sur ses propres tendances suicidaires ou sur quelques autres sinistres épisodes biographiques ${ }^{35}$, il suffit de se souvenir que Benjamin avait perdu sa mère l'année précédente et se remettait à peine de son divorce pour comprendre combien il pouvait être sensible à un récit de ce type. Au-delà de l'anecdote, comme le montre tout particulièrement l'exemple du portrait de Kafka ${ }^{36}$, c'est bien à travers un processus d'identification que se joue, pour Benjamin, l'accès à l'image photographique. Qu'il faille du récit - et le cas échéant du mélodrame - pour enclencher ce processus n'a rien qui puisse surprendre. Au détail près que, loin de s'être " plongé » exclusivement dans l'image, il a fallu à Benjamin le détour complexe d'un texte, voire d'une lecture fautive, pour arriver à la conclusion de l'« ici et maintenant » de la photographie.

Il fallait pourtant ce détour à Benjamin pour qu'il installe la structure temporelle qui seule pouvait induire la conclusion théorique d'un l'au-delà de la représentation. Tuer d'abord, puis ressusciter la fiancée du portrait, inventer un futur au bref instant de la prise de vue pour mieux replonger dans le présent de l'image qu'une vie désormais menacée rend précieux : par une série d'artifices narratifs, le système que Benjamin met en place forme un décalque rigoureux de celui par lequel Proust ramène au présent le passé (pour en faire le seul présent dont on puisse jouir, puisqu'il a cessé de fuir). De même que « les vrais paradis sont les paradis qu'on a perdus $»^{37}$, le présent aperçu par Benjamin dans la photographie n'offre cette qualité, cette plénitude que parce qu'il a précisément cessé de passer : un présent immobile, que l'on peut contempler à loisir, un présent " plein de l'avenir et chargé du passé $»^{38}$, selon la formule de Leibniz qu'évoque la «Petite histoire ${ }^{39}$.

Si l'on rapproche la démarche de Benjamin de celle d'un Roland Barthes, bien des années plus $\operatorname{tard}^{40}$, il semble bien qu'il faille formuler l'hypothèse d'un lien étroit de la photographie avec cette forme du deuil que Freud nomme mélancolie. À la différence de Proust, dont l'expérience demeure enclose dans le champ de sa propre mémoire, Benjamin comme Barthes utilisent leur propre intimité pour approcher et comprendre l'image et $\mathrm{y}$ insuffler du récit. Sans aller jusqu'à parler d'une méthode ou d'une heuristique mélancolique, on doit admettre que ce Fort-da temporel, ce jeu de la mise à distance pour la plus grande proximité ( « unique apparition d'un lointain, aussi proche soit-elle ») témoigne d'une remarquable efficacité. 


\section{NOTES}

1. Walter Benjamin, «Petite histoire de la photographie », ed. et trad. de l'allemand A. Gunthert, Études photographiques, $\mathrm{n}^{\circ}$ 1, 1996, p. 6-39 ; Catherine Perret, « La beauté dans le rétroviseur », La Recherche photographique, $\mathrm{n}^{\circ} 16,1994, \mathrm{p} .74$.

2. "Petite histoire...", op. cit.

3. Helmuth Bossert \& Heinrich Guttmann, Aus der Frühzeit der Photographie. 1840-1870. Francfort/ Main, Societäts-Verlag, 1930.

4. Eugène Atget, Lichtbilder, introd. Camille Recht. Paris-Leipzig, Éd. Jonquières, 1930 ; Heinrich Schwarz, David Octavius Hill. Der Meister der Photographie. Leipzig, Insel V, 1931.

5. La bibliographie allemande de référence (Frank Heidtmann, Hans-Joachim Bresemann \& Rolf H. Krauss, Die deutsche Photoliteratur, 1839-1978. Munich, K. G. Saur, 1980) recense six entrées en la matière entre 1880 et 1931.

6. Josef Maria Eder, Geschichte der Photographie ( $3^{\text {e }}$ éd.). Halle, Wilhelm Knapp, 1905 ; Erich Stenger, Geschichte der Photographie. Berlin, VDI Verlag, 1929.

7. Beaumont Newhall publiera en 1937 la première version de son History of Photography, modèle des histoires de la photographie actuelles. Cf. Marta Braun, "Beaumont Newhall et l'historiographie de la photographie anglophone ", Études photographiques, n 16, 2005, p. 19-31.

8. L'article paraît en trois livraisons successives, les 18 et 25 septembre, et le 2 octobre 1931 (Die Literarische Welt, $7^{\mathrm{e}}$ année, $n^{\circ} 38,1931$ p. 3-4, $n^{\circ} 39$, p. 3-4 et $n^{\circ} 40$, p. 7-8), et comporte environ 40 000 signes.

9. Albert Renger-Patzsch, Die Welt ist schön. Munich, Kurt Wolff, 1928 ; Karl Blossfeldt, Urformen der Kunst. Photographische Pflanzenbilder (intr. K. Nierendorf). Berlin, Ernst Wasmuth, 1928 (rééd. Munich, Harenberg, 1982) ; August Sander, Antlitz der Zeit (intr. A. Döblin). Munich, Langen, 1929 (trad. française par L. Marcou : Visage d'une époque : soixante portraits d'Allemands $d u x^{e}$ siècle. Munich, Paris, Schirmer/Mosel, 1990).

10. Pour une bonne présentation des débats de cette période, voir Olivier Lugon, Le Style documentaire : d'August Sander à Walker Evans, 1920-1945. Paris, Macula, 2001.

11. László Moholy-Nagy, Malerei Photographie Film. Munich, Langen, 1925.

12. Siegfried Kracauer, « Die Photographie » [1927], in Aufsätze. Francfort/Main, Suhrkamp, 1990, vol. 2, p. 83-98 ; Bertolt Brecht, «Über Fotografie » [1928], in Werke, ed. par Werner Hecht. Francfort/Main, Suhrkamp, 1992, vol. 21, p. 264-265 ; Alfred Döblin, « Von Gesichtern, Bildern und ihrer Wahrheit ", in August Sander, Antlitz der Zeit,op. cit., p. 7-15.

13. Précisons que cet engouement ne doit pas conduire à minimiser l'originalité ou le risque que comportait un tel sujet : de fait, on chercherait en vain semblable préoccupation dans les écrits de la très grande majorité des acteurs de l'intelligentsia allemande de l'époque (en 1935, Heidegger choisit par exemple de consacrer sa réflexion aux Souliers de Van Gogh).

14. «Le brouillard qui s'étend sur les commencements de la photographie n'est pas tout à fait aussi épais que celui qui recouvre les débuts de l'imprimerie... ", Walter Benjamin, "Petite histoire de la photographie », op. cit., p. 9.

15. «Ce que l'invention de l'imprimerie fut à la pensée et à son expression parlée, l'invention de la photographie l'a été pour l'apparence et sa représentation iconographique. » (Erich Stenger, Geschichte der Photographie, op. cit., p. 1).

16. Commentant l'historiographie de l'imprimerie, Guy Bechtel résume : «L'objet de ces discours sur les circonstances, outre qu'il fournit à bon prix des alibis et des couvertures idéologiques, est trop fréquemment de présenter l'invention comme toute naturelle, normale, venant à son heure. » (Guy Bechtel, Gutenberg et l'invention de l'imprimerie. Paris, Fayard, 1992, p. 42-43. 
17. Tristan Tzara, «La photographie à l'envers ", in Euvres complètes, éd. Henri Béhar. Paris, Flammarion, 1975, t. 1, p. 415-417 ; traduction de Walter Benjamin : « Die Photographie von der Kehrseite », G-Zeitschrift für Elementare Gestaltung, n 3, 1924, p. 29-30.

18. "Nichts gegen die "Illustrierte" ", in Gesammelte Schriften. Francfort/Main, Suhrkamp, 1982 (cité infra GS), t. IV, vol. 1-2, p. 448-449.

19. « Neues von Blumen », in GS, III, p. 151-153.

20. Cf. Georges Bataille, « Le langage des fleurs », Documents, vol. I, nº 3, 1929, p. 160-168.

21. Pour une bibliographie complète des sources utilisées par Benjamin, voir mon édition critique (Études photographiques, ${ }^{\circ} 1,1996$, p. 38-39).

22. Cf. Alfred Lichtwark, in Fritz Matthies-Masuren, Künstlerische Photographie. Berlin, Marquardt, 1907, p. 4-18.

23. Parmi les textes mentionnés par Benjamin, ceux de Bertold Brecht, Bernard von Brentano, Stefan George, Siegfried Kracauer, Tristan Tzara et Antoine Wiertz proviennent sans aucun doute de lectures n'ayant pas de rapport avecla rédaction de l'article.

24. On trouvera les emprunts non signalés ou la réutilisation de citations issues des sources (comme, par exemple, celle de l'article du Leipziger Anzeiger, empruntée à Max Dauthendey, Der Geist meines Vaters, Munich, Langen, 1925, p. 39-40) dans mon édition critique de la "Petite histoire ", (Études photographiques, $\mathrm{n}^{\circ}$ 1, 1996, op. cit.).

25. Voir notamment Walter Benjamin, «Y. [Die Photographie] », in GS, tome V, vol. 2, p. 824-846 (trad. française par J. Lacoste, Paris, capitale du XIX siècle. Paris, Éd. du Cerf, 1993, p. 685-703).

26. La première date mentionnée est celle du 3 juillet 1839, soit le célèbre discours d'Arago présentant l'invention du daguerréotype à l'Assemblée nationale.

27. Walter Benjamin, « Petite histoire de la photographie », op. cit., p. 7.

28. Heinrich Schwarz, David Octavius Hill..., op. cit., fig. 26. Voir également Sara Stevenson, D. 0. Hill and R. Adamson, Edimbourg, National Gallery of Scotland, 1981, p. 196.

29. Walter Benjamin, "Petite histoire de la photographie", op. cit., p. 9.

30. Helmuth Bossert \& Heinrich Guttmann, Aus der Frühzeit der Photographie..., op. cit., fig. 128.

31. Walter Benjamin, "Petite histoire de la photographie ", op. cit., p. 10-11.

32. Max Dauthendey, Der Geist meines Vaters [1912]. Munich, Langen, 1925, p. 114.

33. Dans certains cas, la psychose puerpérale du post-partum, ou mélancolie puerpérale, peut en effet aboutir au suicide (cf. Henri Ey, Paul Bernard \& Charles Brisset, Manuel de psychiatrie. Paris, Masson, 1978, p. 728-729).

34. Max Dauthendey, ibid., p. 115 (je traduis).

35. En particulier les conditions étranges du décès de sa tante Friederike, cf. Momme Brodersen, Spinne im eigenen Netz. Walter Benjamin, Leben und Werk. Bühl-Moos, Elster Verlag, 1990, p. 25.

36. Benjamin acquiert en 1931 un portrait de Kafka enfant qu'il décrit dans plusieurs textes. On retrouve en particulier dans Enfance berlinoise une version plus détaillée que celle figurant dans la «Petite histoire de la photographie» (op. cit., p. 157-158), qui révèle le processus d'identification avec le sujet de l'image (cf. Walter Benjamin, Berliner Kindheit um Neunzehnhundert, GS, tome IV, vol. 1, p. 261, trad. française par J. Lacoste, Sens unique... Paris, Maurice Nadeau, 1988, p. 68-69).

37. Marcel Proust, À la recherche du temps perdu, éd. P. Clarac \& A. Ferré. Paris, Gallimard, 1954, III, p. 870.

38. Gottfried Wilhelm Leibniz, Nouveaux essais sur l'entendement humain, éd. J. Brunschwig. Paris, GF-Flammarion, 1990, p. 42.

39. Voir supra. Il faudra attendre "Sur quelques thèmes baudelairiens ", paru en 1939, pour que Benjamin établisse et commente le rapprochement de la photographie et de la structure proustienne.

40. Cf. André Gunthert, " Le complexe de Gradiva. Théorie de la photographie, deuil et résurrection », Études photographiques, n²,1997, p. 115-128. 
INDEX

Mots-clés : avant-garde, dépassement de l'art, lecture de l'image, photographie

\section{AUTEUR}

\section{ANDRÉ GUNTHERT}

Historien de l'art, chercheur, éditeur, il est maître de conférences à l'EHESS, où il a créé le Laboratoire d'histoire visuelle contemporaine. Il a fondé et dirige la revue Études photographiques. Specialiste de l'histoire et des pratiques photographiques, ses recherches actuelles analysent les nouveaux usages impliqués par l'images numérique. 